\title{
Validez de dos instrumentos para medir la relación interpersonal de la enfermera con el paciente y su familia en la unidad de cuidado intensivo'
}

\author{
Yaneth Mercedes Parrado Lozano² \\ Ximena Sáenz Montoya ${ }^{3}$ \\ Virginia Inés Soto Lesmes² \\ Sandra Rocío Guáqueta Parada³ \\ Pilar Amaya Rey² \\ Clara Virginia Caro Castillo² \\ Myriam Parra Vargas ${ }^{4}$ \\ Martha Cecilia Triana Restrepo 5
}

doi:10.11144/Javeriana.ie18-1.vimr

Como citar: Parrado Lozano YM, Sáenz Montoya X, Soto Lesmes VI, Guáqueta Parada SR, Amaya Rey P, Caro Castillo CV, Parra Vargas M, Triana Restrepo MC. Validez de dos instrumentos para medir la relación interpersonal de la enfermera con el paciente y su familia en la unidad de cuidado intensivo. Investig Enferm. Imagen Desarr. 2016;18(1): 115-28. http://dx.doi.org/10.11144/Javeriana.ie18-1.vimr

1. Artículo original de investigación. Recibido: 27 de mayo de 2015. Aceptado: 30 de septiembre de 2015.

2. Doctora en Enfermería. Profesora asociada, Universidad Nacional de Colombia. Correo electrónico: ymparradol@unal.edu.co / visotol@unal.edu.co / mcamayad@unal.edu.co / cvcaroc@unal.edu.co

3. Magister en Enfermería. Profesora asociada, Universidad Nacional de Colombia. Correo electrónico: xsaenzm@unal.edu.co / srguaquetap@unal.edu.co

4. Magíster en Educación. Profesora pensionada, Universidad Nacional de Colombia. Correo electrónico mparrav@unal.edu.co.

5. Magíster en Educación. Profesora asociada, Universidad Nacional de Colombia. Correo electrónico mctrianar@unal.edu.co 


\section{Resumen}

Objetivo: Construir, refinar, validar facialmente y de contenido dos instrumentos para medir la relación interpersonal enfermera-familia y enfermera-paciente adulto en la unidad de cuidado intensivo (UCI). Método: Estudio metodológico de abordaje cuantitativo, realizado por fases, para el diseño, validación facial, de contenido y refinamiento de dos instrumentos. Se consideraron los criterios de redacción, gramática, cohesión y coherencia en la validez facial, y la pertinencia y concordancia de cada ítem en la validez de contenido. Resultados: Dos instrumentos; valoración de la relación interpersonal enfermera-paciente en la UCI (VRIEP-UCI) y valoración de la relación interpersonal enfermera-familia o acompañante del paciente en la UCI (VRIEF-UCI), instrumentos a los cuales se les realizó validación facial mediante el juicio de 7 expertas, y de contenido mediante el coeficiente de concordancia de Kappa, superior a 0,50 con una fuerza de concordancia moderada según Landis y koch. Esto permitió ajuste en su diseño y refinamiento con mejor seguridad y exactitud. Conclusión: Esta investigación aporta dos instrumentos diseñados, validados y estructurados para la práctica del cuidado de enfermería en la UCI con población colombiana adulta, que permiten crear evidencia al valorar en el cuidado la relación interpersonal entre la enfermera y el paciente, y entre la enfermera y la familia del paciente.

Palabras clave: relación interpersonal; investigación metodológica en enfermería; enfermería de cuidados críticos

\section{Validity of Two Instruments to Measure Interpersonal Relationship of Nurses with Patients and their Families in the Intensive Care Unit}

\section{Abstract}

Objective: Building, refining, and establishing the face validity of the contents of two instruments to measure the interpersonal nurse-family and nurse-adult patient relationships in the Intensive Care Unit (ICU). Method: Methodological study with a quantitative approach, carried out in stages, for the design, face validity, content validation, and refining of two instruments. We took into account the criteria of grammar, cohesion, and coherence for face validity, and relevance and concordance of each item for the content validation Results: Two instruments; assessment of the interpersonal relationship nurse-patient in the ICU (VRIEP-ICU), and assessment of the interpersonal relationship nurse-family or caretaker of the patient in the ICU (VRIEF - ICU). Face validity was carried out on these instruments by means of the opinion of 7 experts, and content validation was carried out by means of the Kappa concordance quotient, higher than 0.50 , with an almost perfect concordance robustness, according to Landis and Koch. This allowed for adjustments in its design and refining with greater security and precision. Conclusion: This research provides two instruments that were designed, validated, and structured for the practice of nursing care in the ICU, with adult Colombian population, which enables the creation of evidence by assessing during care the interpersonal relationship between the nurse and the patient and the nurse and the family of the patient.

Keywords: interpersonal relationship; methodological research in nursing; critical care nursing 


\section{Validade dos instrumentos para medir a relação interpessoal da enfermeira com o paciente e sua família na unidade de terapia intensiva}

\section{Resumo}

Objetivo: Construção, refinação, validação facial e de conteúdo de dois instrumentos para medir a relação interpessoal enfermeira-família, e enfermeira-paciente adulto, na unidade de terapia intensiva (UCI). Método: Estudo metodológico de abordagem quantitativa, realizado por fases, para o desenho, validação facial, de conteúdo e refinação de dois instrumentos. Consideraram-se os critérios de redação, gramática, coesão e coerência e cada item na validação de conteúdo. Resultados: Dois instrumentos; valoração da relação interpessoal enfermeira-paciente na UTI (VRIEP-UTI) e valoração da relação interpessoal enfermeira-família ou acompanhante do paciente na UTI (VRIEFUTI), instrumentos dos quais se realizou validação facial mediante o julgamento de 7 especialistas, e de conteúdo mediante o coeficiente de concordância Kappa, superior a 0,50 com uma força de concordância quase perfeita segundo Landis e Koch, que permitiu ajustar o desenho e refinação deles com maior certeza e exatidão. Conclusão: Esta pesquisa contribui com dois instrumentos desenhados, validados e estruturados para a prática do cuidado de enfermagem na UTI com população colombiana adulta, que permitem criar evidência ao valorizar no cuidado a relação interpessoal entre a enfermeira e o paciente, e entre a enfermeira e a familia o paciente.

Palavras chave: relação interpessoal; pesquisa metodológica em enfermagem; enfermagem de cuidados críticos 


\section{Introducción}

En nuestro medio no se cuenta con instrumentos o indicadores que admitan crear evidencia empírica para medir en el cuidado la relación interpersonal entre la enfermera y el paciente, y la enfermera y la familia del paciente adulto hospitalizado en la unidad de cuidados intensivos (UCI), en particular de los hospitales universitarios. Con el fin de alcanzar este propósito, se realizó una alianza entre dos grupos de investigación de la Facultad de Enfermería de la Universidad Nacional de Colombia.

Se partió de los productos académicos acumulados, experiencia clínica nutrida por los resultados de investigaciones de cuidado y práctica, riesgo familiar total (RFT) (1), del modelo de cuidado para la práctica y la enseñanza del cuidado crítico (HANC) (2) y de contribuciones de enfermeras de servicio.

El examen de la producción científica en el tema de interés evidencia un vacío de conocimiento en la medición de la relación interpersonal de las enfermeras con las familias y los pacientes adultos hospitalizados en la UCI. Este mismo ejercicio muestra que la comunicación y el trato digno y respetuoso y algunos aspectos del riesgo familiar total no cuentan en nuestro medio con instrumentos que les permita ser medidos.

La identificación de estos vacíos llevó a un equipo de 8 investigadoras a potenciar en una alianza sus conocimientos con el fin de construir y realizar la validación inicial de los instrumentos. Cada vez más, es necesario disponer de instrumentos de medida en el ámbito de la salud que se puedan utilizar en la práctica clínica y en investigación, para garantizar la calidad de su medición es imprescindible que los instrumentos sean sometidos a un proceso de validación (3).

Con frecuencia, las investigaciones metodológicas se centran en los procesos de validez y confiabilidad de los instrumentos (4-6); sin embargo, muchas de estas no ilustran los pasos en el diseño y sustentación del constructo, conceptos, dimensiones y sus respectivos reactivos. Por lo tanto, la presente investigación hace una importante contribución en este sentido. Contar con instrumentos sólidos y pertinentes para el contexto beneficia la prestación del servicio y la satisfacción de los usuarios con el cuidado de enfermería en la red de hospitales de formación de la Universidad Nacional de Colombia.

\section{Objetivo}

Construcción, refinación y validación facial y de contenido de dos instrumentos para medir la relación interpersonal enfermera-familia o acudiente, y enfermera-paciente adulto en la UCI.

\section{Método}

El diseño de los instrumentos se realizó mediante una investigación cuantitativa de abordaje metodológico, organizada en diferentes momentos. La metodología seleccionada permitió diseñar dos instrumentos para medir la relación interpersonal en términos de la interacción de la enfermera con el de enfermería en la UCI. 
Se partió de los hallazgos relacionados con RFT y modelo de cuidado HANC, que establece indicadores de calidad, comunicación y trato humano, que orientan la relación interpersonal entre la enfermera, el paciente y su familia. Así, el trabajo se organizó por fases.

\section{Primera fase. Definición de constructo}

Parte de la revisión y crítica de 157 piezas de investigación, encontradas en las bases de datos: ISI Web, Scopus, Pubmed, Medline, Academic Search Complet, SAGE Journals, American Journal of Critical Care y Cochrane, LILACS y BVS, durante el periodo comprendido entre 2000 y 2013. La literatura en mención abordó términos como comunicación con la familia y el paciente en la UCI, trato digno, cuidado humanizado y relación interpersonal. Posteriormente, la búsqueda se decantó adicionando los siguientes criterios de inclusión: estar disponible en texto completo, términos tesauro enfermeria, que se abordaran en la UCI, que trataran instrumentos (si corresponde), paciente crítico adulto, familia, soporte, necesidades, medición y cuestionario, tanto en inglés como en español.

El determinante o concepto central de la relación interpersonal enfermera-paciente y enfermera-familia, identificado en la producción investigativa revisada, fue la comunicación en la UCI (7-15). Este se constituyó como constructo central.

El constructo para Gras (1980), citado por Abreu (16), es explícito cuando un concepto puede ser observado y medido, y si puede relacionarse con otros conceptos a través de hipótesis, entonces puede utilizársele en la investigación científica y se denomina constructo.

\section{Segunda fase. Definición operacional}

En esta se estandariza el significado, al definir un concepto en términos de las operaciones o procedimientos con que se mide o se determina su presencia (17). También definir operacionalmente una variable es especificar qué actividades $\mathrm{u}$ operaciones debe realizar el investigador para medirla. Estas operaciones se llaman indicadores, y cuando recogen información de la realidad, son capaces de convertirla en datos (18).

Para esta fase, el grupo de investigadoras definió a partir del constructo dos conceptos operacionales: la relación interpersonal enfermera-paciente y enfermera-familia y seis dimensiones.

En varias sesiones de trabajo colectivo se confirmaron las dimensiones que se redujeron para establecer las más significativas y pertinentes en la medición del constructo. Las dimensiones o variables forman parte de los conceptos, y en este caso son la expresión de las habilidades y las capacidades de la enfermera en la UCI de adultos, las cuales son susceptibles de medición para valorar la relación interpersonal de la enfermera con la familia y el paciente adulto que ha sido hospitalizado en la UCI. Surgen en esta 
investigación: la orientación, la información, el trato digno y respetuoso, la empatía, el cuidado participativo y las estrategias comunicativas.

\section{Tercera fase. Construcción de los ítems}

Tiene presente que de la definición operacional de los conceptos se derivó la construcción del conjunto de ítems o reactivos que conformarían cada uno de los dos instrumentos. Este proceso riguroso se dirigió considerando que estos representaran las propiedades de las dimensiones. Las investigadoras trabajaron teniendo en cuenta las bondades del método Delphi, una metodología estructurada para recolectar sistemáticamente juicios de expertos sobre un problema, procesar la información y, a través de recursos estadísticos, construir un acuerdo general de grupo. Permite la transformación durante la investigación de las apreciaciones individuales de los expertos en un juicio colectivo superior (19).

En la redacción de los ítems se consideró: la simplicidad del enunciado, la presencia de un único atributo en el enunciado, la fácil comprensión, el lenguaje claro y sencillo y la redacción en tiempo presente obviando ambigüedades. La sustentación conceptual de los reactivos fue nutrida, además del aporte de las investigadoras, con el trabajo conjunto con estudiantes de pregrado y posgrado.

\section{Cuarta fase. Validación de los instrumentos}

Una vez finalizado el diseño de los instrumentos se dio inicio al proceso de validación facial y de contenido por parte de un equipo de enfermeras con amplia trayectoria en el cuidado de personas adultas en situación crítica de salud, hospitalizadas en la UCI.

La validez facial o de apariencia no supone un concepto estadístico, sino que depende del juicio que los expertos hacen sobre la pertinencia de los ítems (20). Para este caso se consideraron los criterios de gramática, cohesión y coherencia en la redacción. Las pares evaluadoras, siete enfermeras de la práctica seleccionadas de acuerdo con la trayectoria y formación en el área, conformaron el grupo de expertas, en un número impar para evitar posible polarización en los juicios. Estas recibieron una cláusula de confidencialidad, un instructivo, la descripción del contenido de los dos instrumentos y la escala de evaluación.

La validez de contenido valoró la pertinencia y concordancia de cada ítem de los instrumentos. La pertinencia se refiere a identificar si el contenido del ítem tiene correspondencia con el dominio o atributo que se pretende evaluar, y la relevancia identifica si el ítem tiene la importancia suficiente como para evaluar los contenidos definidos. Mitchell, en 1986, manifestaba que el concepto esencial de validez de contenido se refiere a

120 la relevancia y representación del atributo en los items de un instrumento de medición para un propósito particular (21); por lo tanto, la validez de 
contenido se refiere al grado en que el instrumento representa la totalidad del fenómeno que se pretende medir. Este concepto procura que la escala de medición que se diseñe exhiba las siguientes características: induzca a un mínimo de respuestas sesgadas, sea fácil de entender e interpretar, sea fácil de administrar o aplicar en el trabajo de campo y que posea la capacidad de discriminar (22).

Esta investigación es considerada de bajo riesgo según la Resolución 008430 de 1993, Ministerio de Salud de Colombia. De conformidad con esta Resolución, en este estudio prevaleció el respeto por la propiedad intelectual. Desde la Ley 911 de 2004, relacionada con la responsabilidad deontológica del ejercicio de la profesión de enfermería, este estudio se rigió por los principios de veracidad y fidelidad de la información.

\section{Resultados y discusión}

Esta investigación aportó dos instrumentos estructurados a partir del constructo de comunicación: los conceptos operacionales, que son las dimensiones, y los ítems o reactivos, que ayudaron a crear evidencia empírica para valorar en el cuidado la relación interpersonal entre la enfermera y el paciente y entre la enfermera y la familia del paciente adulto hospitalizado en la UCI de hospitales universitarios.

\section{Primera fase}

Los hallazgos de la revisión sistemática de la literatura científica mostraron, en su mayoría, constructos derivados de investigación cualitativa y de revisiones de diverso orden. Estos se contrastaron con los productos de los grupos de investigación de la alianza. Se logra identificar la comunicación en la UCI como un constructo central en la relación interpersonal enfermerapaciente y enfermera-familia, determinante para la satisfacción de las necesidades de estos. Producto del análisis colectivo en torno de los resultados y la experiencia, las investigadoras identificaron dos conceptos centrales: relación enfermera-paciente y relación enfermera-familia.

\section{Segunda fase}

La relación interpersonal de la enfermera con el paciente adulto en la UCI y su familia fue establecida como los conceptos que originaron las dimensiones y, posteriormente, los reactivos que conformaron los indicadores empíricos o instrumentos.

Relación enfermera-paciente: definida como la percepción del paciente para evaluar la relación que recibió de la enfermera cuando estuvo hospitalizado en la UCI, y relación enfermera-familia: percepción del familiar o acompañante del paciente para evaluar la relación que recibió de la enfermera cuando estuvo su paciente hospitalizado en la UCI. Al realizar la definición operacional, quedaron como dimensiones identificadas: 
- Orientación: capacidad de la enfermera para guiar al paciente hospitalizado o su familia sobre las normas y funcionamiento de la UCI, a través de diversos mecanismos.

- Información: capacidad de la enfermera de comunicar al paciente o familia los aspectos relacionados con su situación de salud, evolución y cuidado.

- Trato digno y respetuoso: capacidad de la enfermera para reconocer y valorar positivamente al paciente o familia, como un sujeto de derechos.

- Empatía: capacidad de la enfermera de experimentar la realidad subjetiva del paciente o familia, de tal forma que le permita responder adecuadamente a sus necesidades y les facilite la expresión de sus sentimientos.

- Estrategias comunicativas: capacidad de la enfermera de incluir diferentes maneras de transmitir y recibir mensajes en un contexto particular de interacción con el paciente.

- Cuidado participativo: capacidad de la enfermera de hacer partícipe al familiar para que se involucre de forma voluntaria, gradual y guiada en el cuidado de su paciente, en función de sus posibilidades y la situación de salud.

\section{Tercera fase}

La versión definitiva de los instrumentos se consolidó luego de un amplio proceso que incluyó la construcción, la modificación, la eliminación, la reducción y la determinación de los ítems definitivos, que se presentan en forma de interrogantes con expresiones positivas que permiten medir las dimensiones establecidas, teniendo precaución de no enunciar un juicio previo en la formulación de estos.

Valoración de la relación interpersonal enfermera-paciente en la UCI (VRIEP-UCI). Este instrumento consta de 46 ítems que serán respondidos por el paciente, y será aplicado en el momento del egreso de la UCI o hasta el séptimo día del egreso, con el fin de evaluar la relación que recibió de la enfermera cuando estuvo hospitalizado en la UCI, a partir de las siguientes dimensiones o factores: orientación (7 items), información, trato digno y respetuoso, empatía (10 ítems para cada una de estas dimensiones) y estrategias comunicativas (9 items). Los reactivos cuentan con las siguientes opciones de respuesta: siempre (sí), algunas veces, nunca (no). Las ítems 8, 9, 10, 15, 17, 18 y 27 son de respuesta dicotómica, es decir, sí o no.

Valoración de la relación interpersonal enfermera-familia o acompañante del paciente en la UCI (VRIEF-UCI). Este instrumento consta de 45 items respondidos por el familiar o acompañante del paciente, y será aplicado en el momento del egreso de la UCI o hasta el séptimo día del egreso, con el fin de evaluar la relación que recibió de la enfermera cuando estuvo hospitalizado en la UCI, a partir de las siguientes dimensiones o factores: orientación (7 îtems), información (8 ítems), trato digno y respetuoso, empatía y 
cuidado participativo (10 reactivos para cada una). Los ítems cuentan con las siguientes opciones de respuesta: siempre (sí), algunas veces, nunca (no). Las preguntas $8,10,14,16,17,25,42$ y 44 son de respuesta dicotómica, es decir, sí o no.

\section{Cuarta fase}

Para la validez facial se consideraron los criterios de 1) gramática y cohesión, pues la experta debía evaluar si el ítem estaba formulado de forma correcta en términos de semántica, sintaxis y ortografía, y 2) coherencia, para establecer si el ítem era comprensible y si estaba redactado de forma sencilla que permitiera fácilmente encontrarle sentido a lo que se estaba preguntando.

Las observaciones de cada una de las expertas fueron consignadas para el análisis por parte de las investigadoras en los formatos de validez facial o aparente y de validez de contenido. Esta información sirvió de insumo en la discusión y posterior consenso de las investigadoras para la formulación final de los ítems. En este proceso de construcción colectiva se contó con el acompañamiento, la asesoría y la retroalimentación de un experto en psicometría.

La armonía interjueces o armonización de las medidas de las diferentes observaciones de las expertas mide el grado de concordancia entre los resultados de dos o más observadores al medir la misma variable o acontecimiento (23). Esta armonía se estableció mediante el índice de concordancia de kappa (superior a 0,50), con una fuerza de concordancia moderada, según Landis y Koch (24). Esto permitió el ajuste final en el diseño con seguridad y exactitud, como se muestra en las tablas 1 y 2.

TABLA. 1. Índice de concordancia: VRIEP-UCI

\begin{tabular}{|l|c|c|c|c|c|c|}
\hline \multirow{2}{*}{ İtem } & \multicolumn{2}{|c|}{ Pertinencia } & \multicolumn{2}{c|}{ Relevancia } & \multicolumn{2}{c|}{ Índice de concordancia } \\
\cline { 2 - 7 } & Si & No & Si & No & Pertinencia & Relevancia \\
\hline 1 & 6 & 1 & 7 & - & 0,86 & 1,00 \\
\hline 2 & 7 & - & 7 & - & 1,00 & 1,00 \\
\hline 3 & 6 & 1 & 6 & 1 & 0,86 & 0,86 \\
\hline 4 & 6 & 1 & 5 & 2 & 0,86 & 0,71 \\
\hline 5 & 6 & 1 & 6 & 1 & 0,86 & 0,86 \\
\hline 6 & 6 & 1 & 5 & 2 & 0,86 & 0,71 \\
\hline 7 & 6 & 1 & 7 & - & 0,86 & 1,00 \\
\hline 8 & 6 & 1 & 6 & 1 & 0,86 & 0,86 \\
\hline 9 & 6 & 1 & 7 & - & 0,86 & 1,00 \\
\hline 10 & 6 & 1 & 6 & 1 & 0,86 & 0,86 \\
\hline 11 & 6 & 1 & 6 & 1 & 0,86 & 0,86 \\
\hline 12 & 6 & 1 & 6 & 1 & 0,86 & 0,86 \\
\hline 13 & 6 & 1 & 6 & 1 & 0.86 & 0,86 \\
\hline
\end{tabular}

Continúa 


\begin{tabular}{|c|c|c|c|c|c|c|}
\hline \multirow{2}{*}{ Ítem } & \multicolumn{2}{|c|}{ Pertinencia } & \multicolumn{2}{|c|}{ Relevancia } & \multicolumn{2}{|c|}{ Îndice de concordancia } \\
\hline & Si & No & Si & No & Pertinencia & Relevancia \\
\hline 14 & 7 & - & 7 & - & 1,00 & 1,00 \\
\hline 15 & 7 & - & 5 & 2 & 1,00 & 0,71 \\
\hline 16 & 5 & 2 & 6 & 1 & 0,71 & 0,86 \\
\hline 17 & 6 & 1 & 6 & 1 & 0,86 & 0,86 \\
\hline 18 & 7 & - & 7 & - & 1,00 & 1,00 \\
\hline 19 & 7 & - & 7 & - & 1,00 & 1,00 \\
\hline 20 & 7 & - & 7 & - & 1,00 & 1,00 \\
\hline 21 & 6 & 1 & 7 & - & 0,86 & 1,00 \\
\hline 22 & 7 & - & 7 & - & 1,00 & 1,00 \\
\hline 23 & 6 & 1 & 6 & 1 & 0,86 & 0,86 \\
\hline 24 & 6 & 1 & 7 & - & 0,86 & 1,00 \\
\hline 25 & 7 & - & 7 & - & 1,00 & 1,00 \\
\hline 26 & 6 & 1 & 5 & 2 & 0,86 & 0,71 \\
\hline 27 & 7 & - & 7 & - & 1,00 & 1,00 \\
\hline 28 & 6 & 1 & 6 & 1 & 0,86 & 0,86 \\
\hline 29 & 6 & 1 & 6 & 1 & 0,86 & 0,86 \\
\hline 30 & 7 & - & 6 & 1 & 1,00 & 0,86 \\
\hline 31 & 6 & 1 & 6 & 1 & 0,86 & 0,86 \\
\hline 32 & 6 & 1 & 5 & 2 & 0,86 & 0,71 \\
\hline 33 & 6 & 1 & 6 & 1 & 0,86 & 0,86 \\
\hline 34 & 7 & - & 7 & - & 1,00 & 1,00 \\
\hline 35 & 7 & - & 7 & - & 1,00 & 1,00 \\
\hline 36 & 6 & 1 & 6 & 1 & 0,86 & 0,96 \\
\hline 37 & 6 & 1 & 7 & - & 0,86 & 1,00 \\
\hline 38 & 7 & - & 7 & - & 1,00 & 1,00 \\
\hline 39 & 6 & 1 & 7 & - & 0,86 & 1,00 \\
\hline 40 & 6 & 1 & 7 & - & 0,86 & 1,00 \\
\hline 41 & 7 & 1 & 7 & - & 1,00 & 1,00 \\
\hline 42 & 7 & - & 7 & - & 1,00 & 1,00 \\
\hline 43 & 7 & - & 7 & - & 1,00 & 1,00 \\
\hline 44 & 7 & - & 7 & - & 1,00 & 1,00 \\
\hline 45 & 7 & - & 7 & - & 1,00 & 1,00 \\
\hline 46 & 7 & - & 7 & - & 1,00 & 1,00 \\
\hline 47 & 6 & 1 & 6 & 1 & 0,86 & 0,86 \\
\hline
\end{tabular}

Fuente: Soto V, Guáqueta S, Parrado Y, Sáenz X, Amaya P, Parra M, Caro C, Triana M. Programa de fortalecimiento del cuidado y la práctica de enfermería para personas en situaciones agudas y críticas de salud y su familia, usuarias de la Red de Hospitales 124 Universitarios de la Universidad Nacional de Colombia, sede Bogotá, 2014. 
TABLA 2. Índice de concordancia: VRIEF-UCI

\begin{tabular}{|c|c|c|c|c|c|c|}
\hline \multirow{2}{*}{ Ítem } & \multicolumn{2}{|c|}{ Pertinencia } & \multicolumn{2}{|c|}{ Relevancia } & \multicolumn{2}{|c|}{ Índice de concordancia } \\
\hline & Si & No & Si & No & Pertinencia & Relevancia \\
\hline 1 & 7 & & 7 & - & 1,00 & 1,00 \\
\hline 2 & 6 & 1 & 6 & 1 & 0,86 & 0,86 \\
\hline 3 & 6 & 1 & 6 & 1 & 0,86 & 0,86 \\
\hline 4 & 7 & - & 7 & - & 1,00 & 1,00 \\
\hline 5 & 7 & - & 6 & 1 & 1,00 & 0,86 \\
\hline 6 & 7 & - & 6 & 1 & 1,00 & 0,86 \\
\hline 7 & 6 & 1 & 4 & 3 & 0,86 & 0,57 \\
\hline 8 & 7 & - & 6 & 1 & 1,00 & 0,86 \\
\hline 9 & 6 & 1 & 7 & - & 0,86 & 1,00 \\
\hline 10 & 6 & 1 & 6 & 1 & 0,86 & 0,86 \\
\hline 11 & 6 & 1 & 6 & 1 & 0,86 & 0,86 \\
\hline 12 & 6 & 1 & 6 & 1 & 0,86 & 0,86 \\
\hline 13 & 6 & 1 & 6 & 1 & 0,86 & 0,86 \\
\hline 14 & 6 & 1 & 6 & 1 & 0,86 & 0,86 \\
\hline 15 & 6 & 1 & 6 & 1 & 0,86 & 0,86 \\
\hline 16 & 5 & 2 & 6 & 1 & 0,71 & 0,86 \\
\hline 17 & 5 & 2 & 6 & 1 & 0,71 & 0,86 \\
\hline 18 & 7 & - & 7 & - & 0,86 & 1,00 \\
\hline 19 & 4 & 3 & 5 & 2 & 0,57 & 0,71 \\
\hline 20 & 7 & - & 7 & - & 1,00 & 1,00 \\
\hline 21 & 6 & 1 & 7 & - & 0,86 & 1,00 \\
\hline 22 & 7 & - & 7 & - & 1,00 & 1,00 \\
\hline 23 & 6 & 1 & 6 & 1 & 0,86 & 0,86 \\
\hline 24 & 6 & 1 & 7 & - & 0,86 & 1,00 \\
\hline 25 & 7 & - & 7 & - & 1,00 & 1,00 \\
\hline 26 & 6 & 1 & 6 & 1 & 0,86 & 0,86 \\
\hline 27 & 7 & - & 7 & - & 1,00 & 1,00 \\
\hline 28 & 6 & 1 & 6 & 1 & 0,86 & 0,86 \\
\hline 29 & 7 & - & 7 & - & 1,00 & 1,00 \\
\hline 30 & 6 & 1 & 6 & 1 & 0,86 & 0,86 \\
\hline 31 & 6 & 1 & 6 & 1 & 0,86 & 0,86 \\
\hline 32 & 5 & 2 & 5 & 2 & 1,00 & 0,71 \\
\hline 33 & 7 & - & 7 & - & 1,00 & 1,00 \\
\hline 34 & 7 & - & 7 & - & 1,00 & 1,00 \\
\hline 35 & 7 & - & 6 & 1 & 1,00 & 0,86 \\
\hline 36 & 6 & 1 & 6 & 1 & 0,86 & 0,86 \\
\hline 37 & 6 & 1 & 7 & - & 0,86 & 1,00 \\
\hline 38 & 6 & 1 & 7 & - & 0,86 & 1,00 \\
\hline 39 & 7 & - & 7 & - & 1,00 & 1,00 \\
\hline 40 & 7 & - & 7 & - & 1,00 & 1,00 \\
\hline 41 & 6 & 1 & 7 & - & 0,86 & 1,00 \\
\hline 42 & 7 & - & 7 & - & 1,00 & 1,00 \\
\hline 43 & 7 & - & 7 & - & 1,00 & 1,00 \\
\hline 44 & 7 & - & 7 & - & 1,00 & 1,00 \\
\hline 45 & 6 & 1 & 6 & 1 & 0,86 & 0,86 \\
\hline 46 & 7 & - & 7 & - & 1,00 & 1,00 \\
\hline 47 & 7 & - & 7 & - & 1,00 & 1,00 \\
\hline
\end{tabular}

Fuente: Soto, V., Guáqueta, S., Parrado Y., Sáenz X., Amaya P., Parra M., Caro C., Triana M. Programa de fortalecimiento del cuidado y la práctica de enfermería para personas en situaciones agudas y críticas de salud y su familia, usuarias de la Red de Hospitales Universitarios de la Universidad Nacional de Colombia, sede Bogotá, 2014. 


\section{Conclusiones}

- El proceso investigativo de alianza de grupos de investigación con apoyo y financiación institucional permitió la construcción, la refinación y la validación facial y de contenido de los instrumentos VRIEP-UCI y VRIEF-UCI, para medir la relación interpersonal enfermera-familia y enfermera-paciente adulto, en el cuidado de enfermería en la UCI.

- Presentar detalladamente el abordaje metodológico empleado proporciona información importante a otros investigadores, pues aporta a la construcción de indicadores empíricos que contribuyen a solucionar problemas de la práctica de enfermería y de otras disciplinas de salud, y con ello se mejora la calidad del servicio en escenarios de alta complejidad, como las UCI de los hospitales universitarios.

- El aporte adicional es poder valorar particularmente, desde el paciente, la dimensión de estrategias comunicativas, entendida esta como la capacidad de la enfermera de incluir diferentes maneras de transmitir y recibir mensajes en un ambiente particular de interacción con el paciente, donde ella debe interpretar las manifestaciones de dolor, la interferencia en la comunicación y las necesidades básicas.

\section{Recomendaciones}

- Continuar con la validez de constructo de los instrumentos VRIEPUCI y VRIEF-UCI.

- Utilizar los instrumentos diseñados, que tienen en cuenta los hallazgos de la revisión de tema plasmados en dimensiones como orientación, información, trato digno y respetuoso, empatía, cuidado participativo y estrategias comunicativas tanto desde la visión de la relación de la enfermera con la familia como de la enfermera con el paciente, es certeza de generación de nuevo conocimiento para cualificar la práctica de enfermería, y una contribución metodológica desde su validación.

\section{Conflicto de interés}

Las investigadoras y las autoras del presente artículo manifiestan no tener relaciones o razones que puedan representar un posible conflicto de interés.

\section{Financiación}

Este artículo es resultado de un proceso de investigación Proyecto 14309: Programa de Fortalecimiento del Cuidado y la Práctica de Enfermería para Personas en Situaciones Agudas y Críticas de Salud y su Familia, Usuarias de la Red de Hospitales Universitarios de la Universidad Nacional de Colombia, sede Bogotá. Ficha Quipu: 202010017253. Financiado por la Universidad Nacional de Colombia, sede Bogotá, División de Investigación (DIB).

\section{Referencias}

1. Amaya P. Instrumento de Riesgo Familiar Total RFT: 5-33 manual aspectos teóricos, psicométricos, de estandarización y de aplicación. Bogotá: Unibiblos; 2004. 
2. Parra M, Guáqueta SR, Triana MC. Perspectivas de cuidado en UCI: una visión desde el modelo de cuidado HANC. Bogotá: Kimpres; 2012.

3. Carvajal A, Centeno C, Watson R, Martínez M, Sanz Rubiales Á. ¿Cómo validar un instrumento de medida de la salud? Anales Sis San Navarra [Internet]. 2011 Abr [citado 2015 Mar 23];34(1):63-72. Disponible en: http://scielo.isciii.es/scielo. php?script=sci_arttext\&pid=S1137-66272011000100007\&lng=es.

4. Luna L, Cruz L, Oropeza Y, Medina C, Mejía AM. Diseño y validación de un instrumento para el registro de reacciones adversas a la donación de sangre y sus componentes. Rev Mex Enferm Cardiológica. 2013 May;21(2):50-6.

5. Fernández-Sola C, Granero-Molina J, Mollinedo-Mallea J, Gonzales $\mathrm{MH}$, Aguilera-Manrique G, Ponce Mara L. Desarrollo y validación de un instrumento para la evaluación inicial de enfermería. Rev Esc Enferm USP [Internet]. 2012 Dec [citado 2015 Mar 23];46(6):1415-22. Disponible en: http://www.scielo.br/scielo.php? script=sci_arttext\&pid=S0080-.

6. Echeverría Castro SB et al. Diseño de instrumentos de medición en psicología y sus propiedades psicométricas: competencia metodológica en estudios de psicología [internet]. Sonora: Instituto Tecnológico de Sonora; 2013 [citado 2014 feb]. Disponible en: http://www.itson. $\mathrm{mx} /$ publicaciones.

7. Patak L, Gawlinski A, Fung I, Doering L, Berg J and Henneman EA. Communication boards in critical care: patients' views. Appl Nurs Res. 2006;182-90.

8. Barlem E, Rosenhein DPN, Lunardi VL, Filho VDL. Communication as a tool of humanization of nursing care: experiences in intensive care unit. Rev Eletr Enf. 2008;10(4):1041-9.

9. Winter Y-Y Chui. Stress and coping of Hong Kong Chinese family members during a critical illness. J Clin Nurs. 2008;16:372-81.

10. Boyle DK, Miller PA, Forbes-Thompson SA. Communication and endof-life care in the intensive care unit: patient, family, and clinician outcomes. Crit Care Nurs Q. 2005;28(4):302-16.

11. Endacott R, Kidd T, Chaboyer W, Edington J. Recognition and communication of patient deterioration in a regional hospital: A multimethods study. Aust Crit Care. 2007;20(3):100-5.

12. Alasad J, Ahmad M. Communication with critically ill patients. J Adv Nurs. 2005;50(4):356-62.

13. Phillips C. Use of patient diaries in critical care. Nurs Stand. 2011;26(11):35-43.

14. Finke EH, Light J. Kitko L. A systematic review of the effectiveness of nurse communication with patients with complex communication needs with a focus on the use of augmentative and alternative communication. J Clin Nurs. 2008;17:2102-15.

15. Barlem EL, Rosenhein DPN, Lunardi VL, Filho VDL. Communication as a tool of humanization of nursing care: experiences in intensive care unit. Rev Eletr Enf. 2008;10(4):1041-9. 
16. Abreu JL. Constructos, variables, dimensiones, indicadores y congruencia. Daena. International Journal of Good Conscience. 2012 Nov;7(3):123-30.

17. Sánchez R, Echeverry J. Validación de escalas de medición en salud. Rev Salud Pública. Bogotá. 2004;6(3).

18. Cazau P. Introducción a la investigación en ciencias sociales [internet]. 3a ed. Buenos Aires; 2006. Disponible en: http://alcazaba.unex.es/ asg/400758/MATERIALES/INTRODUCCI\%C3\%93N\%20A\%20LA\%20 INVESTIGACI\%C3\%93N\%20EN\%20CC.SS..pdf.

19. García Valdez M, Suarez M. El método Delphi para la consulta a expertos en la investigación científica. Rev Cubana Salud Pública [internet]. 2013 [citado 2014 jul 23];39(2):253-67. Disponible en: http://scielo.sld. cu/scielo.php?script=sci_arttext\&pid=S0864-34662013000200007\&ln $\mathrm{g}=$ es\&nrm=iso.

20. Sánchez R, Gómez C. Validación de escalas de medición en salud. Rev Salud Pública [internet]. 2004 [citado 2015 mayo 23];6(3):30218. Disponible en: http://www.scielo.org.co/scielo.php?script=sci_.

21. Ding C. Hershberger S. Assessing content validity and content equivalence using structural equation modeling. Structural Equation Modeling: A Multidisciplinary Journal. 2002;9(2):283-97.

22. Aravena PC, Moraga J, Cartes R, Manterola C. Validez y confiabilidad en investigación odontológica. Int J Odontostomat. 2014;8(1):69-75.

23. Gras A. Psicología experimental: un enfoque metodológico. México: Trillas; 1980.

24. Landis JR, Koch GG. The measurement of observer agreement for categorical data. Biometrics. 1977;33:159-74. 